\title{
Gynecological malignancy mimicking a thyroid lymph node metastasis
}

\author{
Simone Pederzoli1 ${ }^{1,2}$, Giorgia Spaggiari ${ }^{1}$, Giuditta Bernardelli ${ }^{3}$, Francesco Mattioli ${ }^{4}$, \\ Cinzia Baldessari ${ }^{5}$, Antonino Maiorana ${ }^{3}$, Vincenzo Rochira ${ }^{1,2}$ and Daniele Santi 1,2 \\ ${ }^{1}$ Unit of Endocrinology, Department of Medical Specialties, Azienda Ospedaliero-Universitaria of Modena, Modena, \\ Italy, 2Unit of Endocrinology, Department of Biomedical, Metabolic and Neural Sciences, ${ }^{3}$ Department of Pathology, \\ University of Modena and Reggio Emilia, Modena, Italy, ${ }^{4}$ Department of Otorhinolaryngology-Head and Neck \\ Surgery, and 5Department of Oncology and Haematology, Azienda Ospedaliero-Universitaria of Modena, \\ Modena, Italy
}

Correspondence should be addressed to D Santi

Email

daniele.santi@unimore.it

\section{Summary}

We present the case of a 69-year-old woman who attended the Endocrinology Unit of Modena for a suspicious lymph node in the left cervical compartment discovered during the follow-up of a recurrent gynecological malignancy. At neck ultrasonography, a thyroid goiter was detected, and the further cytological examination was inconclusive for thyroid nodule and compatible with a localization of an adenocarcinoma with papillary architecture for the lymph node. The histological examination after a left neck dissection confirmed the presence of an intracapsular metastasis of a papillary carcinoma immunohistochemically focally positive for thyroid transcription factor 1 and paired box 8 and negative for thyroglobulin. Subsequently, in the suspicion of a thyroid primitiveness, a total thyroidectomy was performed, revealing an intraparenchymal follicular variant of papillary thyroid carcinoma of $2 \mathrm{~mm}$ in the right lobe. During the follow-up, the appearance of a suspected cervical metastatic lesion led to another neck dissection, histologically compatible with a papillary carcinoma localization, immunohistochemically focally positive for thyroid transcription factor 1 and paired box 8 , and negative for thyroglobulin. The histological revision of surgical specimens suggests the cervical recurrence of the prior gynecological cancer, rather than a thyroid carcinoma metastasis. The case described shows how carefully the cytological, histological and immunoistochemical results must be evaluated in oncological management, considering the whole patient's history.

\section{Learning points:}

- Neck lymph node metastases occasionally originate from anatomically distant primary sites, such as breast, lung, gastro-intestinal tract, genito-urinary tract and CNS.

- Histological and immunohistochemical evaluations play an important role to identify the primary malignant site, although in some cases they could mislead the clinicians.

- A multidisciplinary approach and the evaluation of the whole medical history of the patient are mandatory to guide the diagnostic-therapeutic path and to avoid unnecessary treatments.

\section{Background}

Cervical lymph nodes are a typical site of metastases for malignant tumors originating from the head-neck region (1). Commonly, malignancies most frequently showing cervical lymphatic spread arise from upper aero-digestive tract, thyroid, salivary gland and skin (2). However, neck lymphatic metastases occasionally originate from remote primary sites, such as breast, lung, gastro-intestinal tract, genito-urinary tract and CNS (3). In the management 
of cervical lymph node metastasis, the first mandatory step is the identification of the tumor's primary site, influencing the survival, the clinical outcome and the consequent follow-up. However, since the metastases could be anatomically far from the primitive site (3), it is crucial to improve the diagnostic work-up considering several potential tools. Indeed, cytology, histology and immunohistochemistry could play a considerable role to identify the malignant primary site, both by defining the neoplasm architecture and using tissue-specific markers (4). Secondary disease locations usually share the growth structural architecture with primary tumors, and they could express, in several cases, immunohistochemical markers typical of the native tissue by which the neoplasm originates (5). However, especially in case of poorly differentiated carcinomas or particular histological types (i.e. adenocarcinomas), even the comprehensive evaluation of cyto-histological and immunohistochemical features could be inconclusive (2).

Several immunohistochemical markers are currently considered representative of a specific tissue or organ. However, many of these markers could be expressed in variable percentages also in different types of tumors, originating from different sites (6). This overlap represents the main challenge in the clinical application of immunohistochemistry within the diagnostic work-up of distant metastases.

Here, we present a clinical case in which the histological and immunohistochemical features of a lymph node metastasis oriented the diagnostic pathway to the wrong primary tumor. In particular, a thyroid primitiveness was suspected and supported by immunohistochemical evaluations, although the patient's clinical history suggested a more likely persistence of the prior gynecological malignancies than a new thyroid implication.

\section{Case presentation}

A 69-year-old Caucasian woman attended the Endocrinology Unit of Modena in August 2017 for a multinodular thyroid goiter newly diagnosed. This incidental finding occurred for the detection of a fluorodeoxyglucose PET (FDG-PET)-positive lymph node in the left cervical compartment, subsequently investigated with a neck ultrasound. Indeed, she was in oncological follow-up for an ovarian and uterine mixed cystadenocarcinoma treated with a total laparoscopic hysterectomy and a bilateral oophorectomy with pelvic and lumbar-aortic lymphadenectomy in December
2009. Histologically, the cystadenocarcinoma was mainly endometroid-like with squamous metaplasia areas and papillary features (Fig. 1, panels A and B). The carcinoma affected both ovaries, the myometrium and six on 23 lymph nodes removed. After the surgery, the patient underwent paclitaxel-carboplatin chemotherapy until June 2010, then repeated from February to June 2013. Meanwhile, two further lymph node dissections involving retroperitoneal, lumbar and pelvic regions were performed in September 2011 and in January 2012 for the persistence of multiple node metastases. Finally, the disease persistence was treated with radiotherapy from September to December 2014. Since then, no other signs of persistence/recurrence of neoplastic disease were reported.

Neither familial history nor prior episodes of thyroid diseases emerged during the anamnestic interview. The patient's chronic therapy consisted of acetylsalicylic acid and statin in primary prevention for the presence of nonhemodynamically significant carotid stenosis and eye drops for bilateral glaucoma.

\section{Investigation}

At the first visit, the neck physical examination was unremarkable, in absence of palpable nodules in the thyroid region. While normal values of thyroid-stimulating

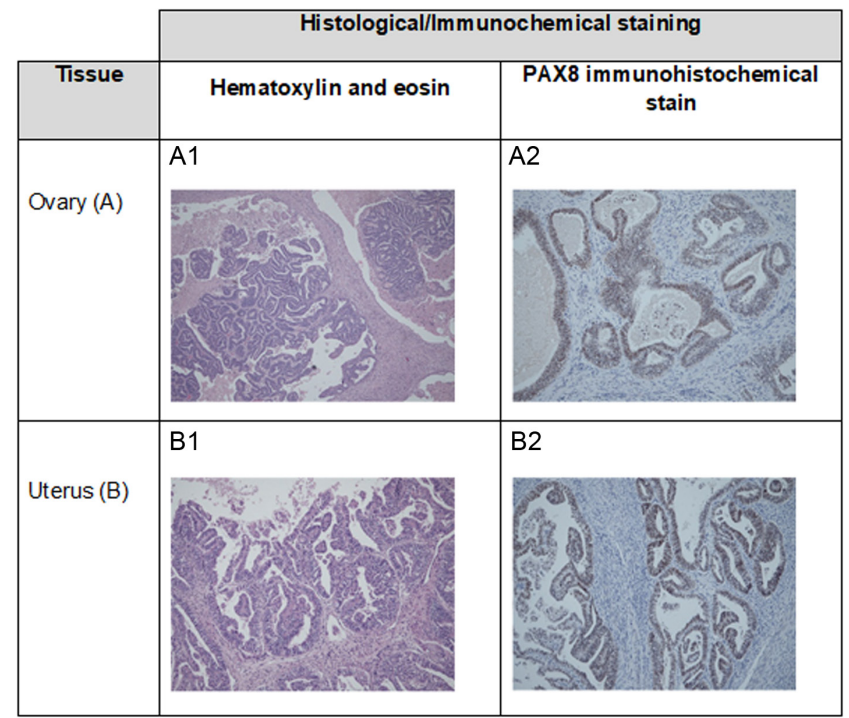

\section{Figure 1}

Histological and immunohistochemical findings during the oncological follow-up of the patient. Pathology preparations of ovary (panel A) and uterus (panel B). Panels 1 refer to hematoxylin and eosin stain, panels 2 refer to immunohistochemical positivity for PAX8. Light microscope images; original magnification: 100×. (PAX8 = paired-box gene 8 ). 
hormone (TSH) were detected $(0.6 \mu \mathrm{IU} / \mathrm{mL}$, reference range $0.27-4.20 \mu \mathrm{IU} / \mathrm{mL}$ ), the sonographic evaluation revealed a thyroid of normal size, with an inhomogeneous echo-structure due to the presence of multiple nodules. In particular, four nodules were detected in the right thyroid lobe and one in the left one. Ultrasound features of the nodules were reported in Table 1 . Three nodules (B, C, D) were classified as intermediate-risk thyroid lesions, while the nodule $\mathrm{A}$ and $\mathrm{E}$ belonged to the highrisk category according to Gharib et al. (7). Moreover, at the third left level of Robbins corresponding to the PET uptake, an inhomogeneous hypoechoic roundish lesion compatible with a suspicious lymph node was detected (Fig. 2). Ultrasound evaluation was performed by Esaote ${ }^{\circledR}$ My Lab25 Gold (Malmesbury, Wiltshire, UK). According to the sonographic characteristics, an ultrasound-guided fine-needle aspiration (FNA) biopsy of nodules A and $\mathrm{E}$ and of the left cervical lesion was performed. The cytological examination of the nodule A highlighted an indeterminate lesion (Tir3a according to the Italian thyroid cytology classification system, 2013) (8), suspected for the follicular nature, while the sample of the nodule $\mathrm{E}$ resulted inadequate (Tir1). The histology of the cervical lymph node was compatible with a localization of adenocarcinoma with papillary features, not otherwise specified (Fig. 3, panel C1). An immunohistochemical positivity for the paired box 8 (PAX8) (Fig. 3, panel C2) and for the thyroid transcription factor1 (TTF1) (Fig. 3, panel C3) suggested a possible thyroid primitiveness of the left cervical lesion. However, the thyroglobulin (Tg) concentration in washout fluid from FNA biopsy (FNA-Tg) was undetectable and the Tg immunohistochemistry was negative on the cytological sample. Since the clinical picture remained unclear, the FNA examination was repeated, confirming the cancerous nature of the cervical lymph node, in absence of evident cytological atypia of the thyroid nodules evaluated.

\section{Treatment}

Following the cytological result, in October 2017 the patient underwent a left neck dissection (levels II-IV of Robbins) at the Unit of Otorhinolaryngology of Modena. The histological examination revealed intracapsular metastasis of papillary carcinoma in two nodes, resulting immunohistochemically diffusely positive for PAX8 (Fig. 3, panel D2), focally positive for TTF1 (Fig. 3, panel D3), negative for Wilms tumor 1 (WT1), estrogen and Tg. The molecular characterization of the metastases revealed a mutation in codon 12 of KRAS (c.35 guanine>alanine),
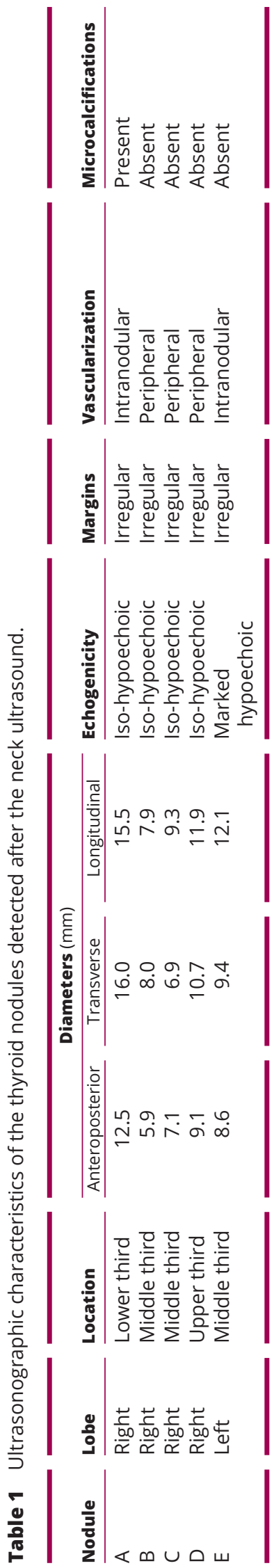


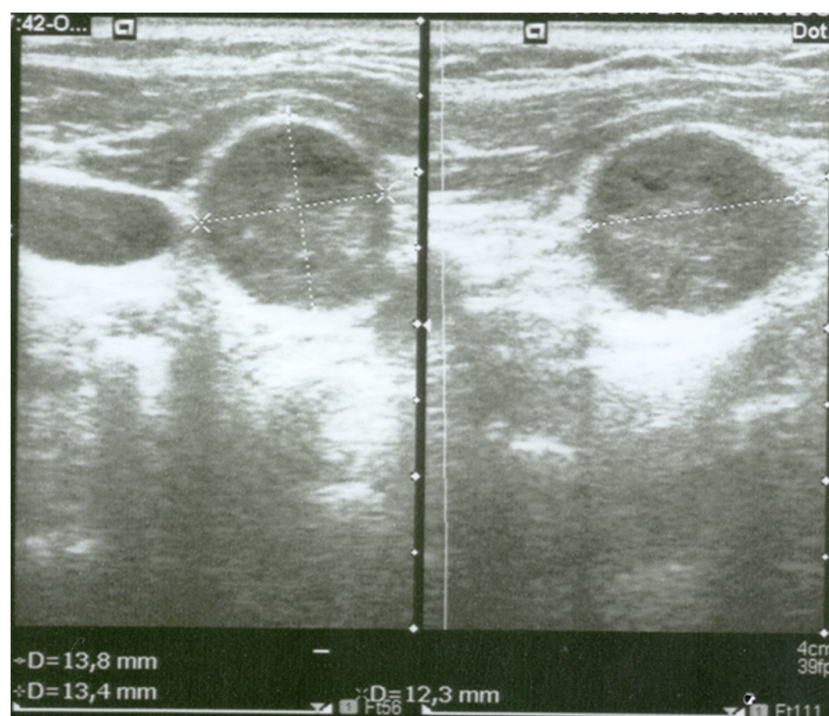

\section{Figure 2}

Ultrasound image of the lymph node on the third left level of Robbins, corresponding to the positron emission tomography (PET) uptake. The lesion appears rounded, markedly hypoechoic and inhomogeneous with increased vascularization. Under the image, the anteroposterior, transverse and longitudinal diameter were reported.

while no alterations were detected on BRAF and NRAS genes. Since PAX8 and TTF1 could also be expressed in different kind of tumors (Table 2), such as thyroid, ovarian and uterine ones, there was no certainty about the primitive origin of the neoplasia. Nevertheless, in the suspicion of a thyroid-native malignancy, a total thyroidectomy associated with bilateral central neck dissection was performed. After the surgery, the patient started the levothyroxine replacement therapy at the posology of $75 \mu \mathrm{g}$ daily and a calcium-vitamin integration for the occurrence of a post-surgical hypoparathyroidism. The thyroid histological examination revealed an intraparenchymal follicular variant of papillary thyroid carcinoma of $2 \mathrm{~mm}$ in the right lobe (pT1as NOa MO stage 1 according to the $8^{\text {th }}$ edition of the American Joint Committee on Cancer staging system) (9). Considering the low stage of the thyroid disease, a further ablative treatment with iodine-131 was deemed unnecessary, and a biochemical and sonographic follow-up was proposed.

\section{Outcome and follow-up}

After 7 months, the patient referred the appearance of a further palpable cervical swelling at the left $\mathrm{Vb}$ level of Robbins. A neck ultrasound scan showed a vascularized nodule of $28 \mathrm{~mm}$ of longitudinal diameter with a mixed component, both fluid and solid, in the context of the sternocleidomastoid muscle. In the suspect of an umpteenth disease recurrence, a PET-computerized tomography (CT) was performed, confirming a pathological glucose uptake in correspondence of the detected lesion (standardized uptake value 20.7).

After the surgical removal of the mass, the histological examination revealed a localization of papillary carcinoma without any evidence of nodal residues, immunohistochemically diffusely positive for PAX8, focally positive for TTF1 and p53, and negative for estrogen receptor and cancer antigen 125 (Fig. 3, panels E). Considering (i) the overall oncologic history, (ii) the very low probability of metastatic disease due to the very small primary thyroid cancer, and (iii) the immunophenotype of metastases, which was also similar to the prior gynecological tumor profile, a pathological revision of the case was carried out, suggesting a more likely ovarian/uterine cystadenocarcinoma persistence of disease, 'exonerating' the thyroid primitiveness.

Considering the aggressive disease behavior, the seeming unique metastatic site, as well as the good general conditions of the patient, an adjuvant radiotherapy on the left cervical region was further proposed and then performed in January 2019.

At the last follow-up evaluation in September 2019, there were no signs of relapse for both malignancies, that is, the ovarian/uterine adenocarcinoma and the thyroid microcarcinoma. In particular, the biochemical assessment documented $\mathrm{Tg}$ serum levels close to zero (0.2 $\mathrm{ng} / \mathrm{mL}$ ), in absence of anti-Tg antibodies, and a negative ultrasound neck scan. Moreover, a normal phospho-calcic profile was detected suggesting a recovery of parathyroid functionality, allowing the calcium-vitamin therapy discontinuation.

\section{Discussion}

The case described represents an emblematic example of the limits of histological and immunohistochemical evaluations in oncological pathology. In our patient, the TTF1 and PAX8 positivity at the immunohistochemical analysis of the neck metastatic lymph node focused the clinical attention to the thyroid gland. Indeed, the combined TTF1 and PAX8 positivity could be identified in thyroid carcinomas (10). TTF1 belongs to the NKx2 family of homeodomain transcription factors and mediates the transcription of the $T g$ (11). Although an immunohistochemical positivity for TTF1 and Tg is detectable in the majority of thyroid tumors with similar sensitivity, TTF1 is more expressed in poorly differentiated carcinomas and related metastasis compared to $\mathrm{Tg}$ (11). In 


\begin{tabular}{|c|c|c|c|}
\hline & \multicolumn{3}{|c|}{ Histological/lmm unochemical staining } \\
\hline Tissue & $\begin{array}{l}\text { Hematoxylin and } \\
\text { eosin }\end{array}$ & $\begin{array}{c}\text { PAX8 } \\
\text { immunohistochemical } \\
\text { stain }\end{array}$ & $\begin{array}{c}\text { TTF1 } \\
\text { immunohistochemical } \\
\text { stain }\end{array}$ \\
\hline $\begin{array}{l}\text { First } \\
\text { cervical } \\
\text { lymph } \\
\text { node } \\
\text { metastasis } \\
\text { (biopsy) } \\
\text { (C) }\end{array}$ & 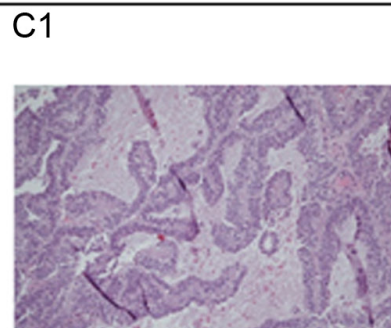 & 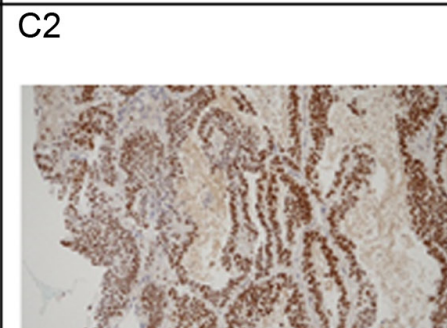 & is \\
\hline $\begin{array}{l}\text { First } \\
\text { cervical } \\
\text { lymph } \\
\text { node } \\
\text { metastasis } \\
\text { (histology) } \\
\text { (D) }\end{array}$ & D1 & D2 & D3 \\
\hline $\begin{array}{l}\text { Second } \\
\text { cervical } \\
\text { lymph } \\
\text { node } \\
\text { metastasis } \\
\text { (histology) } \\
\text { (E) }\end{array}$ & 3 & E2 & E3 \\
\hline
\end{tabular}

\section{Figure 3}

Histological and immunohistochemical findings during the oncological follow-up of the patient. Pathology preparations of first cervical lymph node metastasis (biopsy: panel C, histology: panel D) and second cervical lymph node metastasis (panel E) were reported. Panels 1 refer to hematoxylin and eosin stain, panels 2 refer to immunohistochemical positivity for PAX8, and panels 3 refer to immunohistochemical positivity for TTF1. Light microscope images; original magnification: 100×. (PAX8 = paired-box gene 8; TTF1 = thyroid transcription factor 1).

our case, we detected a TTF1+/Tg- in the first metastasis, thus the possible existence of a poorly differentiated thyroid cancer could not be a priori excluded. On the other hand, since PAX8 is a fundamental transcription factor for organogenesis of the thyroid gland, kidney, and Mullerian-derived organs (12), its expression is not surprisingly detectable in a relevant percentage of tumor originating from thyroid, renal-urinary tract, breast and female and male reproductive system (13). Thus, the PAX8 positivity detected in our case in the first lymph node metastasis could not rule out a primitive thyroid cancer. However, both TTF1 and PAX8 immunohistochemical positivity is not pathognomonic of thyroid primitiveness, since their expression could be present in variable percentage in several tumors (Table 2) (14). This challenge could be further complicated by the histologic 
Table 2 Expression of thyroid transcription factor 1 (TTF1) and paired-box gene 8 (PAX8) in different types of human cancer.

\begin{tabular}{|c|c|c|}
\hline & Expression & Cancer type \\
\hline \multicolumn{3}{|l|}{ TTF1 } \\
\hline & \multirow[t]{7}{*}{$100 \%$} & Glioma \\
\hline & & Thyroid cancer \\
\hline & & Carcinoid \\
\hline & & Colorectal cancer \\
\hline & & Head and neck cancer \\
\hline & & Testis cancer \\
\hline & & Breast cancer \\
\hline & \multirow[t]{2}{*}{$90 \%$} & Prostate cancer \\
\hline & & Cervical cancer \\
\hline & \multirow[t]{3}{*}{$80 \%$} & Lung cancer \\
\hline & & Stomach cancer \\
\hline & & Skin cancer \\
\hline & \multirow[t]{2}{*}{$78 \%$} & Urothelial cancer \\
\hline & & Melanoma \\
\hline & \multirow[t]{2}{*}{$70 \%$} & Ovarian cancer \\
\hline & & Pancreatic cancer \\
\hline & $60 \%$ & Liver cancer \\
\hline & \multirow[t]{2}{*}{$50 \%$} & Lymphoma \\
\hline & & Endometrial caner \\
\hline & $45 \%$ & Renal canner \\
\hline \multicolumn{3}{|l|}{ PAX8 } \\
\hline & $79-90 \%$ & Thyroid cancer \\
\hline & $88-100 \%$ & Renal cancer \\
\hline & $56-74 \%$ & Carcinoid \\
\hline & $79-100 \%$ & Ovarian cancer (primary) \\
\hline & $70-96 \%$ & Ovarian cancer (metastatic) \\
\hline
\end{tabular}

appearance. Indeed, a micro-papillary histologic structure could be common in thyroid cancers and also in ovarian/ endometrial carcinomas (15). In our case, the discovery of an adenocarcinoma in the cervical lymph node dissected with the peculiar immunohistochemistry (i.e. TTF1+/ Tg-/PAX8+) could be explained by either a thyroid or ovarian/endometrial primitiveness. Considering that the papillary-featured adenocarcinoma represents the most common histological type of thyroid malignancies (16) and the concomitant presence of thyroid nodules with undetermined cytology, a possible thyroid primitiveness was suspected. In contrast, FNA-Tg was negative on the cervical lymph node metastasis. However, a false negativity in FNA-Tg could be detected in thyroid-malignant lymph nodes in several cases (17).

Considering all previous histological and immunohistochemical features of the cervical lymph node, a thyroid dissection was performed, revealing a follicular variant of intraparenchymal micro-papillary thyroid carcinoma at the histological examination. However, the further medical history and the subsequent pathological revision has classified this microcarcinoma as an incidental finding. This condition is not infrequent for thyroid carcinomas, particularly for microcarcinomas, since the fortuitous post-surgery histological identification of these malignancies is described in large series of patients thyroidectomized for benign thyroid diseases (18). Indeed, thyroid tumors present generally a low aggressiveness and a slow growth, requiring often several years to spread to locoregional lymph nodes and/or to other organs. As a confirmation, the clinician attitude at treating this kind of tumors is actually debated in the scientific community and an increasingly conservative approach is proposed (18).

Cervical lymph nodes metastases typically originate from head and neck malignancies, due to anatomical proximity and to the structure of the lymphatic drainage pathways from these sites (2). However, anatomicallydistant primary sites are described (2). Although the physio-pathological mechanism of atypical lymphatic spreads remains unclear, the correct identification of the primitive malignancy exerts a significant impact on the patient's management (2). Considering gynecological malignancies, even if the main lymphatic dissemination route involves the retroperitoneal pelvic and para-aortic lymph nodes, cases of further extension to the cervical nodes are reported (19), especially for cervical and ovarian cancers, rather than endometrial ones (2). For anatomical reasons, left-sided neck metastases are more frequent (20), but cervical lymph node involvement from gynecological malignancies remains rare and only few cases are reported in the literature (21).

In the case described, a controversial cyto-histological profile together with an atypical cervical localization of a gynecological malignancy misled the clinicians, causing a probably inappropriate thyroid surgical removal. Although the incidental finding of a thyroid microcarcinoma partially reduces the inadequacy of the procedure, the patient has been exposed to thyroid surgery complications, such as hypoparathyroidism and recurrent laryngeal nerve injury (22), and to the need for chronic levothyroxine replacement therapy. Thus, in presence of an histologically documented thyroid microcarcinoma and cervical lymph nodes metastases other primary cancer should be considered according to patient's medical history, because the metastases of these non-thyroidal adenocarcinomas may mask a metastasis due to thyroid cancer.

In conclusion, cyto-histological analyses and immunohistochemical evaluations represent fundamental tools in clinical oncology management. However, an adequate approach cannot disregard the evaluation of the 
previous medical history, together with the findings derived from physical, biochemical and radiological examinations. In a controversial clinical presentation, a multidisciplinary approach is more than ever mandatory to guide the diagnostic-therapeutic path, avoiding unnecessary treatments and the possible related complications.

\section{Declaration of interest}

The authors declare that there is no conflict of interest that could be perceived as prejudicing the impartiality of the research reported.

\section{Funding}

This work did not receive any specific grant from any funding agency in the public, commercial or not-for-profit sector.

\section{Patient consent}

Written informed consent for publication of her clinical details was obtained from the patient.

\section{Author contribution statement}

$S P, G S$ and D S wrote the manuscript and were involved in the endocrine care of the patient. $\mathrm{V} R$ revised the manuscript. $\mathrm{F} M$ performed the neck surgery on the patient. $G B$ and $A M$ provided histological and immunohistochemical images and performed the anatomo-pathological analyses during the patient management. C B followed the patient within the oncological team.

\section{References}

1 Pilborough AE, Lambert DW \& Khurram SA. Extranodal extension in oral cancer: a role for the nodal microenvironment? Journal of Oral Pathology and Medicine 201948 863-870. (https://doi.org/10.1111/ jop.12870)

2 Lopez F, Rodrigo JP, Silver CE, Haigentz M, Jr, Bishop JA, Strojan P, Hartl DM, Bradley PJ, Mendenhall WM, Suarez C, et al. Cervical lymph node metastases from remote primary tumor sites. Head and Neck 201638 (Supplement 1) E2374-E2385. (https://doi. org/10.1002/hed.24344)

3 Sanchez-Iglesias JL, Capote S, Cubo-Abert M, Carbonell-Socias M, Cabrera S, Illan-Hernandez L, Perez-Benavente MA, Monreal-Clua S \& Gil-Moreno A. A giant superinfected uterine angioleiomyoma with distant septic metastases: an extremely rare presentation of a benign process and a systematic review of the literature. Archives of Gynecology and Obstetrics 2019300 841-847. (https://doi. org/10.1007/s00404-019-05267-w)

4 Miguel AFP, Mello FW, Melo G \& Rivero ERC. Association between immunohistochemical expression of matrix metalloproteinases and metastasis in oral squamous cell carcinoma: systematic review and meta-analysis. Head and Neck 202042 569-584. (https://doi. org/10.1002/hed.26009)

5 Torlakovic EE. Fit-for-purpose immunohistochemical biomarkers Endocrine Pathology 201829 199-205. (https://doi.org/10.1007/ s12022-018-9529-4)
6 Kalampokas E, Payne F, Nomikos A \& Gurumurthy M. An update on the use of immunohistochemistry and molecular pathology in the diagnosis of pre-invasive and malignant lesions in gynecological oncology. Gynecologic Oncology 2018150 378-386. (https://doi. org/10.1016/j.ygyno.2018.05.023)

7 Gharib H, Papini E, Garber JR, Duick DS, Harrell RM, Hegedus L, Paschke R, Valcavi R \& Vitti P. American Association of Clinical Endocrinologists, American College of Endocrinology, and Associazione Medici Endocrinologi Medical Guidelines for Clinical Practice for the diagnosis and management of thyroid nodules - 2016 update. Endocrine Practice 201622 1-60. (https://doi. org/10.4158/EP161208.GL)

8 Nardi F, Basolo F, Crescenzi A, Fadda G, Frasoldati A, Orlandi F, Palombini L, Papini E, Zini M, Pontecorvi A, et al. Italian consensus for the classification and reporting of thyroid cytology. Journal of Endocrinological Investigation 201437 593-599. (https://doi. org/10.1007/s40618-014-0062-0)

9 Zanoni DK, Patel SG \& Shah JP. Changes in the 8th edition of the American Joint Committee on Cancer (AJCC) staging of head and neck cancer: rationale and Implications. Current Oncology Reports 201921 52. (https://doi.org/10.1007/s11912-019-0799-x)

10 Nikiforov YE. Molecular diagnostics of thyroid tumors. Archives of Pathology and Laboratory Medicine 2011135 569-577. (https://doi. org/10.1043/2010-0664-RAIR.1)

11 Bejarano PA, Nikiforov YE, Swenson ES \& Biddinger PW. Thyroid transcription factor-1, thyroglobulin, cytokeratin 7, and cytokeratin 20 in thyroid neoplasms. Applied Immunohistochemistry and Molecular Morphology 20008 189-194. (https://doi.org/10.1097/00129039200009000-00004)

12 Tacha D, Zhou D \& Cheng L. Expression of PAX8 in normal and neoplastic tissues: a comprehensive immunohistochemical study. Applied Immunohistochemistry and Molecular Morphology 201119 293-299. (https://doi.org/10.1097/PAI.0b013e3182025f66)

13 Xiang L \& Kong B. PAX8 is a novel marker for differentiating between various types of tumor, particularly ovarian epithelial carcinomas. Oncology Letters 20135 735-738. (https://doi. org/10.3892/ol.2013.1121)

14 Zhang Y, Yang J, Zhang M, Meng Z, Song W, Yang L, Li L, Wang D \& Shi T. Thyroid follicular carcinoma-like renal tumor: a case report and literature review. Medicine 201897 e10815. (https://doi. org/10.1097/MD.0000000000010815)

15 Kaku T, Ogawa S, Kawano Y, Ohishi Y, Kobayashi H, Hirakawa T \& Nakano H. Histological classification of ovarian cancer. Medical Electron Microscopy 200336 9-17. (https://doi.org/10.1007/ s007950300002)

16 Baloch ZW \& LiVolsi VA. Cytologic and architectural mimics of papillary thyroid carcinoma. Diagnostic challenges in fine-needle aspiration and surgical pathology specimens. American Journal of Clinical Pathology 2006125 (Supplement) S135-S144. (https://doi. org/10.1309/YY72M308WPEKL1YY)

17 Moon JH, Kim YI, Lim JA, Choi HS, Cho SW, Kim KW, Park HJ, Paeng JC, Park YJ, Yi KH, et al. Thyroglobulin in washout fluid from lymph node fine-needle aspiration biopsy in papillary thyroid cancer: large-scale validation of the cutoff value to determine malignancy and evaluation of discrepant results. Journal of Clinical Endocrinology and Metabolism 201398 1061-1068. (https://doi. org/10.1210/jc.2012-3291)

18 Reinke R, Mathiesen JS, Larsen SR, Hahn CH, Pedersen HB, Bentzen J, Schytte S, Godballe C, Londero SC \& A study from The Danish Thyroid Cancer Group - DATHYRCA (part of the DAHANCA organization). Incidental and non-incidental papillary thyroid microcarcinoma in Denmark 1996-2015: a national study on incidence, outcome and thoughts on active surveillance. Cancer Epidemiology 201960 46-50. (https://doi.org/10.1016/j. canep.2019.03.011) 
Endocrinology

Diabetes \& Metabolism

CASE REPORTS
S Pederzoli and others

Neck lymph node metastasis:

not always thyroid
ID: 20-0055; January 2021

DOI: 10.1530/EDM-20-0055
19 Chen CW, Torng PL, Chen CL \& Chen CA. Clinical features and outcomes of neck lymphatic metastasis in ovarian epithelial carcinoma. World Journal of Surgical Oncology 201311 255. (https:// doi.org/10.1186/1477-7819-11-255)

20 Diddle AW. Carcinoma of the cervix uteri with metastases to the neck. Cancer 197229 453-455. (https://doi.org/10.1002/10970142(197202)29:2<453::aid-cncr2820290230>3.0.co;2-7)

21 Kojima M, Yokoyama J, Ito S, Ohba S, Fujimaki M \& Ikeda K. Impact of middle and lower jugular neck dissection on supraclavicular lymph node metastasis from endometrial carcinoma. World Journal of Surgical Oncology 201210 143. (https://doi.org/10.1186/1477-781910-143)

22 Bove A, Panaccio P, Palone G, Esposito L, Marino L \& Bongarzoni G. Impact of the new guidelines of the American Thyroid Association on the treatment of the differentiated thyroid tumor in an Italian Center with medium-high volume thyroid surgery. BMC Surgery 201918 (Supplement 1) 127. (https://doi.org/10.1186/s12893-0180462-8)

Received in final form 31 August 2020

Accepted 16 December 2020 